\title{
Autologous Peripheral Blood Mononuclear Cell Recognition of Autologous Proliferating Tumor Cells in the Context of a Patient-Specific Vaccine Trial
}

\author{
A. N. Cornforth, G. Lee, and R. O. Dillman \\ Cell Biology Laboratory, Hoag Cancer Center, Newport Beach, CA 92663, USA \\ Correspondence should be addressed to A. N. Cornforth, andrew.cornforth@hoaghospital.org \\ Received 1 September 2010; Revised 10 February 2011; Accepted 27 February 2011 \\ Academic Editor: Theresa L. Whiteside
}

Copyright ( 2011 A. N. Cornforth et al. This is an open access article distributed under the Creative Commons Attribution License, which permits unrestricted use, distribution, and reproduction in any medium, provided the original work is properly cited.

Metastatic melanoma patients who were treated with patient-specific vaccines consisting of dendritic cells loaded with autologous tumor cells had a 5-year survival of over 50\%. Enzyme-linked immunospot (ELISPOT) has been used to detect antigen reactive T cells as a means of determining immune response. We wished to determine whether IFN-gamma secretion in an ELISPOT assay was prognostic or predictive for survival following treatment. Peripheral blood mononuclear cells (PBMCs) collected at weeks 0 and 4 were evaluated by ELISPOT assay for response to autologous tumor cells. Overall, there was slight increase in the number of tumor reactive lymphocytes from week 0 to week 4 . Using $>5$ spots $/ 100 \mathrm{~K}$ PBMC as the cutoff, a log-rank analysis revealed only a slight statistical significance in overall survival for patients who lacked tumor reactive PBMCs at week 4 . The sensitivity of ELISPOT in the context of patient-specific cellular vaccines is unclear.

\section{Introduction}

Metastatic melanoma is generally considered to be incurable. Immunotherapy is a promising alternative treatment to chemotherapy, but challenges remain in determining response to therapy. Clinical trials that use adoptive immunotherapy regimens need surrogate markers to measure responsiveness. Currently, measuring changes in the level of serum cytokines by the enzyme-linked immunosorbant assay (ELISA) does not measure specific antigen responses. A novel-cell-based immune monitoring assay like the enzyme-linked immunosorbant spot (ELISPOT) assay may be a more precise and quantifiable measure of immune response.

Attempts to measure changes in serum cytokines involved in immune response (i.e., IL-10 and IL-12) by ELISA yielded no detectable levels in patients treated with the dendritic cell-based vaccine (unpublished observations). In light of this, we chose to use the ELISPOT assay because it is able to measure antigen-specific responses in very small subsets of effector cell populations. Such assays have been touted as a measure of whether a vaccine is enhancing immunity and as possible prognostic and predictive markers for melanoma patients receiving cancer vaccines made up of melanoma-specific peptides such as MART-1, gp100 and tyrosinase [1]. Cytokines that are secreted by immune cells in response to antigenic stimulation are rapidly captured by antibodies before they are diluted in the supernatant, captured by receptors of other cells, or degraded. The colored spot end product typically represents an individual cytokineproducing cell that can be counted. This allows ELISPOT assays to detect immune reactive cells that may be present at a frequency of less than 1/100,000 peripheral blood mononuclear cells. ELISPOT has proved to be a reliable assay for lymphocyte recognition of peptide antigens based on the secretion of cytokines such as interferon gamma.

We recently observed some remarkably long periods of progression-free survival and an encouraging 5-year survival rate of $50 \%$, in patients with metastatic melanoma who were treated with patient-specific vaccines consisting of autologous dendritic cells loaded with interferon-gammatreated and irradiated autologous tumor cells that had 
been proliferating in culture [2]. The vaccinations were given once a week for three weeks and once a month for five months. The cell-based vaccine was administered in granulocyte macrophage-colony stimulating factor (GMCSF) and injected subcutaneously.

In this series of experiments, we used an ELISPOT assay as a metric for postvaccination immune augmentation. The sensitivity of such assays in the context of our clinical trial is unclear since we were immunizing with antigens derived from tumor cells without awareness of which tumorassociated antigens might be most important in the immune response. In this case, we could not use an ELISPOT tetramer assay which employs the use of specific peptide to detect antigen specific $\mathrm{T}$ cells because we were not focusing on a single specific antigen [3]. We therefore had to rely on the release of gamma interferon from a population of autologous lymphocytes based on their exposure to cryopreserved autologous tumor cells that had been proliferating in vitro. We hypothesized that these cells were the best representatives for monitoring immune response for each patient since they represent the patient's own tumor cells and subsequently may contain all of the available tumor associated antigens.

\section{Materials and Methods}

2.1. Patients. Melanoma patients with recurrent or metastatic disease were enrolled in a phase I/II clinical trial [2]. All patients had to be off any immunosuppressive therapy for at least 4 weeks and meet ECOG (Eastern Cooperative Oncology Group) performance levels of 0-2 at the time of initial treatment. At the beginning of treatment, patients were tested for anergy to common recall antigens using available tests for Candida, trichophyton, and mumps but anergic patients were not denied treatment. Those patients with brain metastases not currently receiving pharmacologic doses of corticosteroids with therapy controlled tumors were eligible for enrollment. A total of 54 patients were clinically evaluable but only 42 were included in this study.

2.2. Autologous Dendritic Cell Generation. Dendritic cells (DCs) were generated by plastic adherence method as previously described $[4,5]$. Briefly, peripheral blood mononuclear cells were isolated by ficoll-hypaque (GE Healthcare, Buckinghamshire, United Kingdom) density gradient separation from apheresis collections. The adherent populations were then incubated in AIM-V supplemented with $1000 \mathrm{IU} / \mathrm{mL}$ each of IL-4 (CellGenix, Freiburg, Germany) and GM-CSF (Invitrogen, Grand Island, NY) for 6 days prior to loading with autologous tumor cells.

2.3. Autologous Tumor Cell Line Generation. Pure melanoma tumor cell lines (defined as containing less than 5\% fibroblasts), generated using techniques previously described [68], were incubated with $1000 \mathrm{IU} / \mathrm{mL}$ of IFN- $\gamma$ (InterMune, Brisbane, CA) for 72 hours, irradiated with 100 Gy from a cesium source, and cryopreserved [9]. The tumor cells were recovered from cryopreservation, washed $3 \mathrm{x}$ with PBS, and then added to the in vitro cultivated DCs and incubated for $\sim 24$ hours. The antigen loaded DC were harvested by gentle scraping with a rubber policeman and cryopreserved at equal amounts in 9-11 aliquots.

2.4. Treatment Regimen. Aliquots of dendritic cells loaded with tumor cells were thawed in a $37^{\circ} \mathrm{C}$ water bath, washed $2 \mathrm{x}$ with AIM-V, combined with $500 \mathrm{ug} / \mathrm{mL}$ GM-CSF in saline and subcutaneously injected at weeks 1, 2, 3, 8, 12, 16, 20, and 24. Peripheral blood mononuclear cells (PBMCs) were obtained from peripheral blood samples at weeks 0 and 4 and were cryopreserved after separation of red blood cells by ficoll-hypaque density centrifugation.

2.5. IFN- $\gamma$ Enzyme-Linked Immunospot (ELISPOT) Assay. Using BD ELISPOT Human IFN- $\gamma$ ELISPOT Kit (BD Pharmingen, San Diego, Calif), thawed peripheral blood mononuclear cells (PBMCs) were rested for 24 hours and then cultured in four replicates at $2.0 \times 10^{5}$ viable cells with or without $4.0 \times 10^{5}$ viable autologous tumor cells per well in AIM-V in nitrocellulose backed 96 well plates. The PBMC: ATC ratio and incubation time were chosen based on work by Malyguine et al. [10]. Controls consisted of PBMCs stimulated with a combined cocktail of $3000 \mathrm{IU} / \mathrm{mL}$ IL-2, $2 \mu \mathrm{g} / \mathrm{mL}$ PHA, and $5 \mu \mathrm{g} / \mathrm{mL}$ OKT3 as a positive control, PBMCs or tumor cells only and AIM-V medium only as background and negative controls, respectively. After 48 hour incubation, plates were washed and stained as per manufacturer's instructions. Briefly, plates were washed twice with $200 \mu \mathrm{L} /$ well deionized water and then three times with $200 \mu \mathrm{L} /$ well Wash Buffer (provided by kit). $100 \mu \mathrm{L} /$ well of $1 \mu \mathrm{g} / \mathrm{mL}$ mouse antihuman IFN- $\gamma$-biotinylated mAb (provided by kit) in assay diluent was added and the plates were incubated for 2 hours at room temperature. The plates were then rinsed, incubated with streptavidin-HRP solution for 1 hour and developed with aminoethylcarbazole (AEC) peroxidase substrate solution provided with the kits. The plates were then counted on an ImmunoSpot instrument (Cellular Technology Ltd. Cleveland, Ohio).

2.6. Statistical Analysis. Significant differences were defined by $P$-value $\leq .05$ using Student's $t$-test of two-tailed, two samples of equal variance. Log-rank calculations were used to determine whether there was a significant difference in survival curves. Progression-free and overall survival times were determined by time in months from first injection to last followup or patient expiration.

\section{Results}

The detection of antigen-specific peripheral blood $\mathrm{T}$ lymphocytes was determined by IFN- $\gamma$ Enzyme-linked Immunospot (ELISPOT) assay using autologous tumor cells as the antigen source. Controls consisted of PBMC only (unstimulated), PBMCs exposed to a cytokine cocktail to induce the expression of IFN- $\gamma$ (nonspecific stimulation), tumor cells only or media only (Figure 1). Neither the tumor cell only nor media controls resulted in any spotting 
TABLE 1: Significant changes in tumor reactive $\mathrm{T}$ cells as measured by ELISPOT in patients receiving DC-based immunotherapy for metastatic melanoma.

\begin{tabular}{lc}
\hline Change in relative number of spots & $\begin{array}{c}\text { Week } 4 \text { versus week } 0 \\
(N=42)\end{array}$ \\
\hline Increase $P \leq .05$ & $7(16.6 \%)$ \\
Decrease $P \leq .05$ & $4(9.5 \%)$ \\
No change $P \geq .05$ & $31(73.8 \%)$ \\
\hline
\end{tabular}

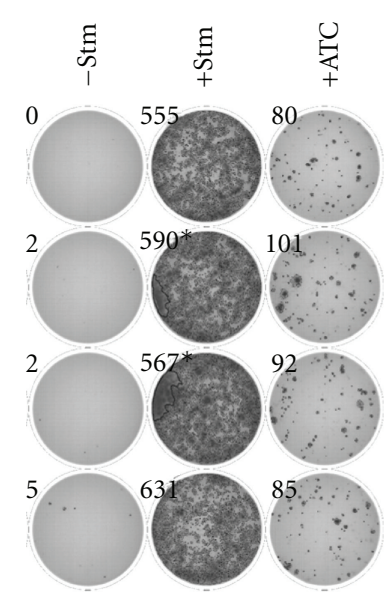

FIGURE 1: Peripheral blood lymphocytes were placed in the wells of ELISPOT plates in quadruplicate under three separate conditions: unstimulated ( - Stm), stimulated with $3000 \mathrm{IU} / \mathrm{mL}$ IL-2, $2 \mu \mathrm{g} / \mathrm{mL}$ PHA, and $5 \mu \mathrm{g} / \mathrm{mL}$ OKT3 ( + Stm), or combined with autologous tumor cells at a ratio of $1: 2$ (+ATC). After 48 hours, IFN$\gamma$ secretion was determined as per manufacturer's instructions. Numbers indicate spots counted by automated counter.

activity in all wells examined (data not shown). The levels of ELISPOT spotting were adjusted for nonspecific (unstimulated) IFN- $\gamma$ secreting lymphocytes and the results are shown in Figure 2.

Out of 54 patients treated on the vaccine study, 42 were evaluable at weeks 0 and 4 for the ELISPOT testing. Although there was no significant change in the average number of tumor reactive PBMCs by week 4, there was trend toward slightly higher frequencies (week 0: $27.1 \pm$ 6.9 spots $/ 100 \mathrm{~K}$ and week 4: $30.9 \pm 7.4$ spots $/ 100 \mathrm{~K}, P=$ $.708)$. Only $7 / 42(16.6 \%)$ demonstrated a significant increase $(P \leq .05)$ in tumor reactive lymphocytes at week 4 versus week 0 . Additionally, 4/42 (9.5\%) demonstrated a significant decrease in tumor reactive lymphocytes at week 4 versus week 0 (Table 1). No correlations could be made with progression-free or overall survival in either group with significant changes in the number of spots between week 0 and week 4 either increasing or decreasing (data not shown).

Moodie et al. reported that an acceptable ELISPOT signal-to-noise ratio of $2: 1$ to $3: 1$ results in a detection limit of 4 to 6 spots $/ 1 \times 10^{5}$ PBMC, respectively, for determining response to the applied antigen [11]. We applied this methodology to our assay and adjusted for any background

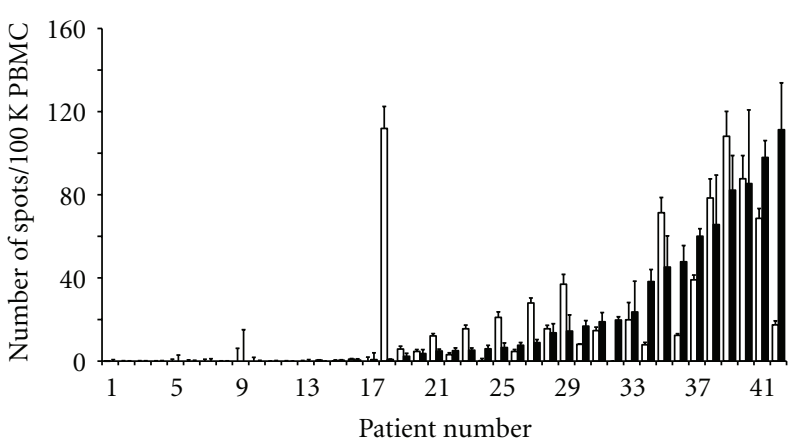

FIGURE 2: Results of induction of IFN- $\gamma$ secreting T lymphocytes detected by ELISPOT in response to autologous tumors cells after DC-based immunotherapy for melanoma. Peripheral blood lymphocytes collected (PBMC) at week 0 (open bars) and week 4 (filled bars) were coincubated with purified autologous tumor cells at a ratio of $1: 2$, respectively, and incubated for 48 hours. Data shown is sorted by average number of spots/100 K PMBC \pm SD. Dashed line approximates the 5 spots/100 K PBMC threshold.

by subtracting the activity of the nonstimulated PBMCs (Figure 2). At week 0 or baseline, 20/42 (47.6\%) showed a significant number of spots $\left(>5\right.$ spots $\left./ 1 \times 10^{5} \mathrm{PBMC}\right)$ in response to ATC. A similar result was seen at week 4, with 20/42 (47.6\%) patients demonstrating response to their autologous tumor cells. Many of the same patients had measurable responses at both weeks (17/20).

Patients were then divided into two cohorts based upon whether they demonstrated greater than or less than 5 spots per 100,000 PBMC in response to autologous tumor cells as means of defining responders versus nonresponders at that time in their treatment schedule. Table 2 describes the patient clinical characteristics at the time of treatment for these cohorts. Log-rank tests were calculated and Kaplan-Meier plots generated for progression-free and overall survival at either week 0 or week 4 . Baseline activity of tumor reactive lymphocytes at week 0 failed to correspond to either progression-free or overall survival. But at week 4, tumor reactivity correlated unfavorably to overall survival but was not associated with progression-free survival (Figure 3). A log-rank comparison of the 17 patients that had sustained tumor reactive lymphocytes ( $>5$ spots $/ 100 \mathrm{~K}$ PBMC at both week 0 and week 4) did not indicate an association with progression-free or overall survival $(P=.177$ and $P=.156$, resp.).

Although it appears that patients in the $>5$ spots $/ 100 \mathrm{~K}$ PBMC had significantly higher lactose dehydrogenase (LDH) serum values at both week 0 and week 4 (Table 2), no significant correlation to clinical performance was found based solely on LDH values $(P=.197$ and $P=.263$, PFS and OS, resp.). Additionally, the number of ECOG 1 and 2 patients in the $>5$ spots $/ 100 \mathrm{~K}$ PBMC is higher $(8$ versus 4 in the $<5$ spots $/ 100 \mathrm{~K}$ PBMC group) which may influence the survival results since it was noted that patients who have higher ECOG scores tend to not perform as well clinically. 
TABLE 2: Comparison of patient characteristics in the two cohorts with ELISPOT results less than or greater than 5 spots/100 K PBMC in response to autologous tumor cells at week 0 and week 4 after receiving dendritic-cell-based antimelanoma therapy.

\begin{tabular}{|c|c|c|c|c|c|c|}
\hline & & All & Week 0 ( $<5$ spots $)$ & Week 0 (>5 spots) & Week 4 (<5 spots) & Week 4 ( $>5$ spots) \\
\hline \multirow{2}{*}{ Sex } & M & 26 & 14 & 12 & 15 & 11 \\
\hline & $\mathrm{F}$ & 16 & 8 & 8 & 7 & 9 \\
\hline Age (years) & & $49.3 \pm 14.1$ & $46.0 \pm 14.8$ & $52.9 \pm 12.8$ & $46.7 \pm 14.3$ & $52.0 \pm 13.8$ \\
\hline \multirow{2}{*}{ Disease status } & $\mathrm{M} 1 \mathrm{a} / \mathrm{b}$ & 20 & 7 & 13 & 7 & 13 \\
\hline & M1c & 22 & 15 & 7 & 15 & 7 \\
\hline Anergy & & 11 & 6 & 5 & 6 & 5 \\
\hline \multirow{9}{*}{ Prior treatment } & RT & 6 & 3 & 3 & 3 & 3 \\
\hline & Chemo & 12 & 6 & 6 & 5 & 7 \\
\hline & IL-2 & 10 & 7 & 3 & 6 & 4 \\
\hline & IFN-alpha & 15 & 9 & 6 & 8 & 7 \\
\hline & GM-CSF & 18 & 9 & 9 & 9 & 9 \\
\hline & Vaccine & 6 & 3 & 3 & 3 & 3 \\
\hline & BCG & 2 & 1 & 1 & 0 & 2 \\
\hline & $\mathrm{mAb}$ & 1 & 1 & 0 & 1 & 0 \\
\hline & Biochemo & 16 & 11 & 5 & 11 & 5 \\
\hline \multirow{4}{*}{ DC phenotype } & $\%$ CD80 & $38.6 \pm 15.1$ & $33.5 \pm 15.2$ & $44.4 \pm 13.1$ & $34.9 \pm 14.7$ & $42.8 \pm 14.8$ \\
\hline & \% CD83 & $10.9 \pm 7.4$ & $12.2 \pm 7.0$ & $9.5 \pm 7.7$ & $12.5 \pm 7.1$ & $9.1 \pm 7.5$ \\
\hline & \% CD86 & $82.5 \pm 9.3$ & $78.1 \pm 13.5$ & $69.6 \pm 19.6$ & $77.3 \pm 13.0$ & $70.4 \pm 20.3$ \\
\hline & $\%$ CD11c & $90.6 \pm 7.3$ & $89.3 \pm 7.9$ & $92.0 \pm 6.4$ & $88.8 \pm 7.7$ & $92.7 \pm 6.3$ \\
\hline \multirow{2}{*}{ Doses 1-3 } & Cell no. $\times 10^{6}$ & $16.3 \pm 7.0$ & $16.2 \pm 6.8$ & $16.4 \pm 7.2$ & $16.1 \pm 7.2$ & $16.4 \pm 6.7$ \\
\hline & $\%$ Viability & $80.4 \pm 9.6$ & $79.0 \pm 9.6$ & $82.0 \pm 9.1$ & $81.0 \pm 9.6$ & $79.8 \pm 9.8$ \\
\hline \multirow{2}{*}{ DTH } & DTH+ (week 0) & 0 & 0 & 0 & 0 & 0 \\
\hline & DTH+ (week 4) & 4 ( 2 eqv $)$ & 2 (2 eqv) & 2 & 1 (1 eqv) & 3 \\
\hline \multirow{3}{*}{ ECOG status } & 0 & 30 & 18 & 12 & 18 & 12 \\
\hline & 1 & 10 & 3 & 7 & 3 & 7 \\
\hline & 2 & 2 & 1 & 1 & 1 & 1 \\
\hline \multirow{2}{*}{ serum LDH $(\mathrm{ng} / \mathrm{mL})$} & LDH (week 0) & $578.6 \pm 391.3$ & $453.2 \pm 93.7$ & $716.6^{*} \pm 531.4$ & $487.6 \pm 162.3$ & $678.7 \pm 530.2$ \\
\hline & LDH (week 4) & $594.6 \pm 504.9$ & $441.7 \pm 92.9$ & $762.9^{*} \pm 695.5$ & $496.1 \pm 321.6$ & $703.1 * \pm 642.0$ \\
\hline
\end{tabular}

Each value is average \pm SD where applicable. M1a: distant skin, lymph nodes, M1b: lung, and M1c: brain, liver or other visceral organs. RT: radiotherapy, Chemo: chemotherapy (temodar, cisplatin, vinblasin, tamoxifen, etc.), Vaccine: allogeneic tumor cell/lysate or peptide vaccines, BCG: bacillus calmette guerin, mAb: MDX-010, Biochemo: IL-2 \pm IFN plus chemotherapy, DTH: delayed type hypersensitivity, eqv: equivocal result, LDH: lactose dehydrogenase. ${ }^{*} P \leq .05$ compared to $<5$ spots/100 K PBMC.

\section{Discussion}

Attempts to find a reliable surrogate for monitoring of immune response during the course of immune-based therapies for cancer continues to be a challenge. The generation of cytotoxic T lymphocytes (CTL) can be used as a measure of the effectiveness of antigen presentation by dendritic cells, but only in a minority of cases does antigen-specific CTL activity correlate with clinical outcome [12].

The ELISPOT assay is a convenient method to monitor immune response since the cells used in the assay can be cryopreserved during the course of treatment and assayed together at a later date thus avoiding plate-toplate variability. Samples handled in this way, proved to be reliably accurate and there appeared to be no adverse functional effects on the peripheral blood lymphocytes due to cryopreservation as previously reported [13]. The use of whole tumor cells as the antigen source, even at significantly high concentration $\left(4 \times 10^{5} /\right.$ well $)$, did not contribute to significant background in the ELISPOT assay, indicating that even a heterogeneous population of autologous tumor cells can be used in this assay.

Other investigators have reported immune responses in the context of ELISPOT assays which correlated with either disease-free survival or overall survival $[1,14]$ but in those cases, the antigens used to induce immunity were specific peptides plus GM-CSF without dendritic cells. In studies that have involved the use of dendritic cells, the correlation between ELISPOT results and survival have been mixed [15] and only a few reports have been published that use autologous tumor cells as the antigen source to measure immune recognition which were found not to correspond to outcome [16]. In addition, the extent of disease burden of patients enrolled in a study in which 


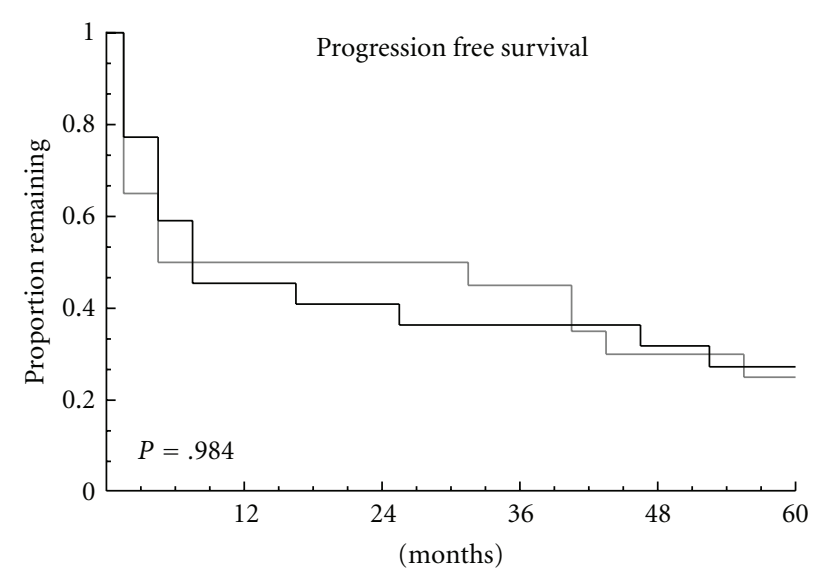

(a)

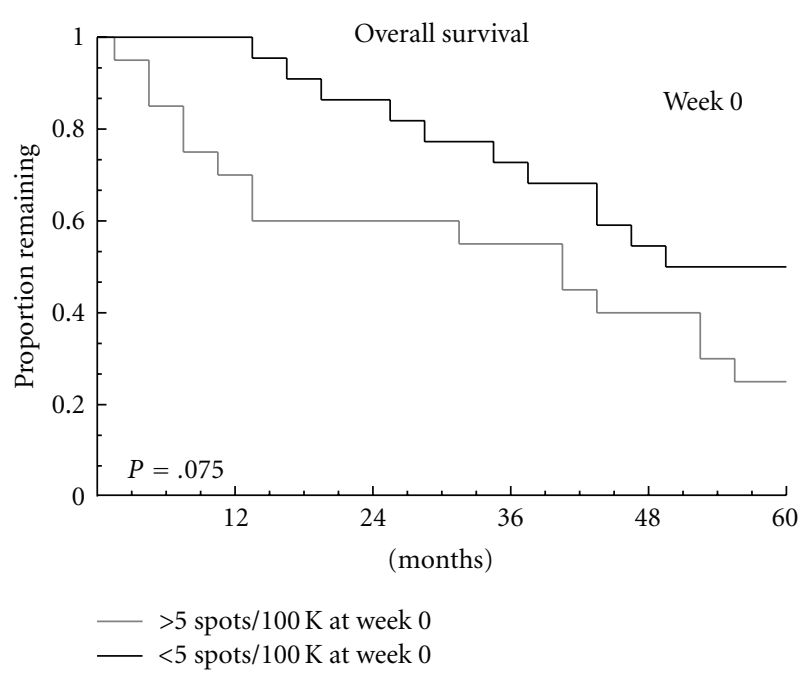

(c)

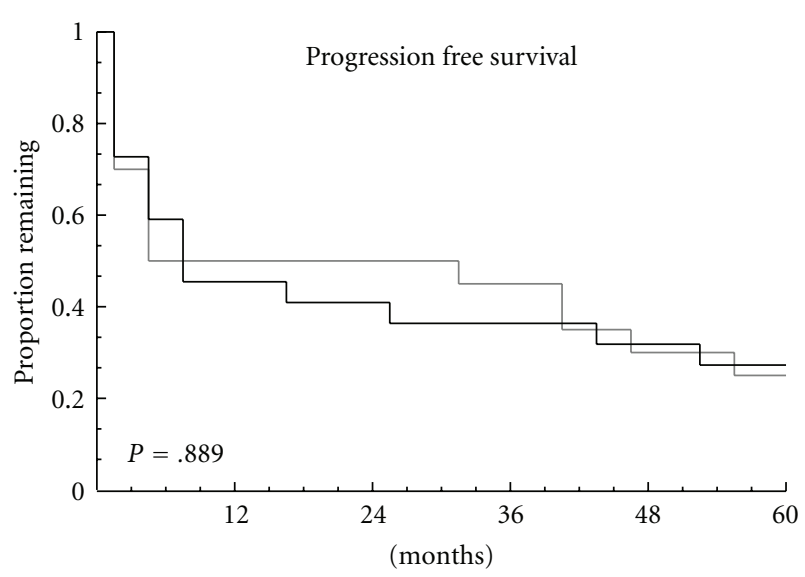

(b)

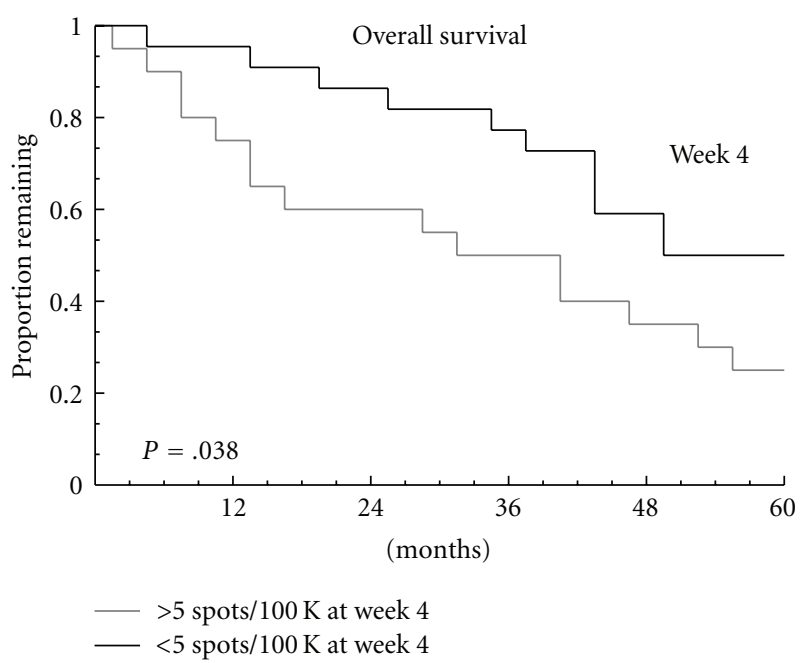

(d)

FIgURE 3: The induction of IFN- $\gamma$ secreting lymphocytes in response to autologous tumor cells and correlation to either progression-free or overall survival at either week 0 or week 4 . Patient cohorts were grouped according to whether they had an average of greater than $(>)$ or less than $(<) 5$ spots/100 K PBMC per well observed at week 0 and week 4 . Sample sizes: Week $0, N=20$ for $>5$ spots/well and 22 for $<5$ spots/well, and for week $4, N=20$ for $>5$ spots/well and 22 for $<5$ spots/well. Log-rank testing was used to determine $P$ values.

immune monitoring was conducted was also a contributing factor in measurable responses. Patients with heavy disease burden were reported to be less responsive immunologically to therapy [17]. However, in our study it appeared that many of the patients who had a higher ECOG performance scores (ECOG 1 or 2) retained their ability to respond to their autologous tumors cells. It should be noted however that patients in this study group with ECOG scores of 1 or 2 had significantly lower progression-free and overall survival than those who had ECOG scores of $0(P=.002$ and .001 , resp.). This contribution may explain why there appears to be a significant difference in overall survival in patients who have $>5$ spots $/ 100 \mathrm{~K}$ PBMC as indicated in Figure 3.

However, the observation of the correlation of lack of response to autologous tumor cells and improved overall survival is counter intuitive. The reason for that observation remains unclear but a recent publication by our laboratory showed a relationship between the induction of apoptosis in response to interferon-gamma by tumor cells and poorer clinical performance [18]. The use of apoptotic cells in immunotherapy has been associated with the induction of tolerance in some cases [19]. It is possible that patients who responded to their autologous tumor cells by releasing interferon-gamma in the ELISPOT assay may also be inducing tolerance in situ (i.e., at the site of disease). Tumor infiltrating lymphocytes encountering tumor cells may be releasing interferon-gamma which in turn induces tolerance through apoptosis of tumor cells and phagocytosis of resident dendritic cells.

Overall, the results are in line with previous reports dealing with complex immune responses but further investigations will be necessary to determine what immune monitoring assay can be employed in this setting. 


\section{References}

[1] J. M. Kirkwood, S. Lee, S. Moschos et al., "Immunogenicity and antitumor effects of vaccination with peptide vaccine +/- granulocyte-monocyte colony-stimulating factor and/or IFIN- $\alpha 2 b$ in advanced metastatic melanoma: Eastern cooperative oncology group phase II trial E1696," Clinical Cancer Research, vol. 15, no. 4, pp. 1443-1451, 2009.

[2] R. O. Dillman, S. R. Selvan, P. M. Schiltz et al., "Phase II trial of dendritic cells loaded with antigens from self-renewing, proliferating autologous tumor cells as patient-specific antitumor vaccines in patients with metastatic melanoma: final report," Cancer Biotherapy and Radiopharmaceuticals, vol. 24, no. 3, pp. 311-319, 2009.

[3] P. Schiltz and R. Dillman, "A theoretical approach for Tlymphocyte monitoring of autologous cancer vaccine therapy using autologous HLA-class I and HLA-class II constructs," Cancer Biotherapy and Radiopharmaceuticals, vol. 19, no. 4, pp. 405-410, 2004.

[4] D. Choi, M. Perrin, S. Hoffmann et al., "Dendritic cellbased vaccines in the setting of peripheral blood stem cell transplantation: CD34+ cell-depleted mobilized peripheral blood can serve as a source of potent dendritic cells," Clinical Cancer Research, vol. 4, no. 11, pp. 2709-2716, 1998.

[5] T. Luft, K. C. Pang, E. Thomas et al., "A serum-free culture model for studying the differentiation of human dendritic cells from adult CD34+ progenitor cells," Experimental Hematology, vol. 26, no. 6, pp. 489-500, 1998.

[6] R. O. Dillman, S. K. Nayak, and L. Beutel, "Establishing in vitro cultures of autologous tumor cells for use in active specific immunotherapy," Journal of Immunotherapy, vol. 14, no. 1, pp. 65-69, 1993.

[7] R. O. Dillman, L. D. Beutel, A. N. Cornforth, and S. K. Nayak, "Short-term tumor cell lines from renal cell carcinoma for use as autologous tumor cell vaccines in the treatment of kidney cancer," Cancer Biotherapy and Radiopharmaceuticals, vol. 15, no. 2, pp. 161-168, 2000.

[8] S. R. Selvan, D. J. Carbonell, A. W. Fowler, A. R. Beatty, M. H. Ravindranath, and R. O. Dillman, "Establishment of stable cell lines for personalized melanoma cell vaccine," Melanoma Research, vol. 20, no. 4, pp. 280-292, 2010.

[9] S. R. Selvan, R. O. Dillman, A. W. Fowler, D. J. Carbonell, and M. H. Ravindranath, "Monitoring response to treatment in melanoma patients: potential of a serum glycomic marker," International Journal of Cancer, vol. 122, no. 6, pp. 1374-1383, 2008.

[10] A. Malyguine, S. L. Strobl, K. A. Shafer-Weaver et al., "A modified human ELISPOT assay to defect specific responses to primary tumor cell targets," Journal of Translational Medicine, vol. 2, article 9, 2004.

[11] Z. Moodie, L. Price, C. Gouttefangeas et al., "Response definition criteria for ELISPOT assays revisited," Cancer Immunology, Immunotherapy, vol. 59, no. 10, pp. 1489-1501, 2010.

[12] W. J. Lesterhuis, I. J. M. de Vries, G. J. Adema, and C. J. A. Punt, "Dendritic cell-based vaccines in cancer immunotherapy: an update on clinical and immunological results," Annals of Oncology, vol. 15, supplement 4, pp. iv145-iv151, 2004.

[13] H. T. Maecker, J. Moon, S. Bhatia et al., "Impact of cryopreservation on tetramer, cytokine flow cytometry, and ELISPOT," BMC Immunology, vol. 6, article 17, 2005.

[14] C. L. Slingluff, G. R. Petroni, K. A. Chianese-Bullock et al., "Immunologic and clinical outcomes of a randomized phase II trial of two multipeptide vaccines for melanoma in the adjuvant setting," Clinical Cancer Research, vol. 13, no. 21, pp. 6386-6395, 2007.

[15] A. Escobar, M. López, A. Serrano et al., "Dendritic cell immunizations alone or combined with low doses of interleukin2 induce specific immune responses in melanoma patients," Clinical and Experimental Immunology, vol. 142, no. 3, pp. 555-568, 2005.

[16] B. G. Redman, E. C. Alfred, J. Whitfield et al., "Phase Ib trial assessing autologous, tumor-pulsed dendritic cells as a vaccine administered with or without IL-2 in patients with metastatic melanoma," Journal of Immunotherapy, vol. 31, no. 6, pp. 591598, 2008.

[17] J. A. Marshall, T. H. Forster, D. M. Purdie et al., "Immunological characteristics correlating with clinical response to immunotherapy in patients with advanced metastatic melanoma," Immunology and Cell Biology, vol. 84, no. 3, pp. 295-302, 2006.

[18] A. N. Cornforth, A. W. Fowler, D. J. Carbonell, and R. O. Dillman, "Resistance to the proapoptotic effects of interferongamma on melanoma cells used in patient-specific dendritic cell immunotherapy is associated with improved overall survival," Cancer Immunology, Immunotherapy, vol. 60, no. 1, pp. 123-131, 2010.

[19] A. E. Morelli and A. T. Larregina, "Apoptotic cell-based therapies against transplant rejection: role of recipient's dendritic cells," Apoptosis, vol. 15, pp. 1083-1097, 2010. 


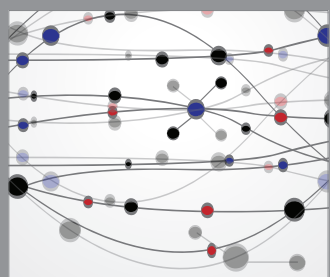

The Scientific World Journal
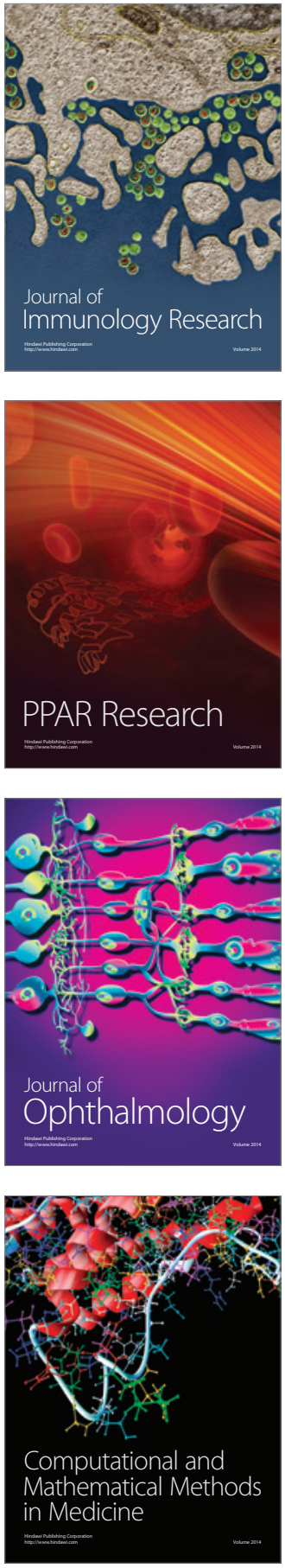

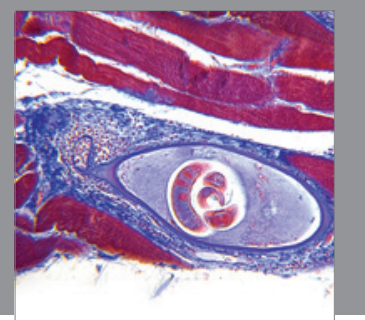

Gastroenterology

Research and Practice
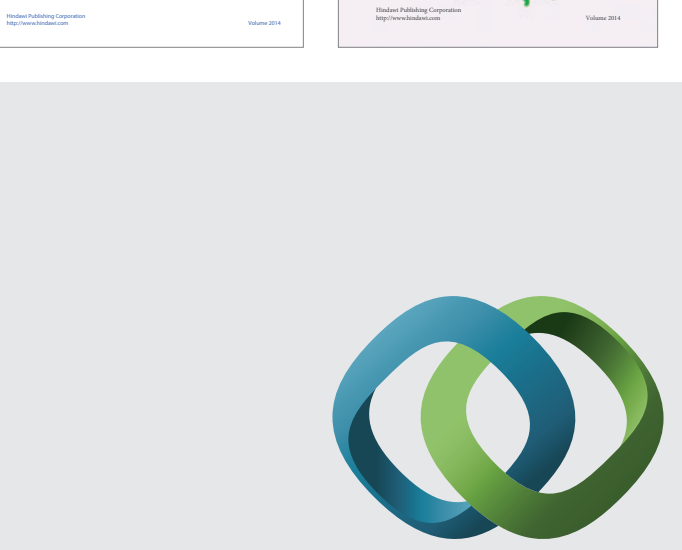

\section{Hindawi}

Submit your manuscripts at

http://www.hindawi.com
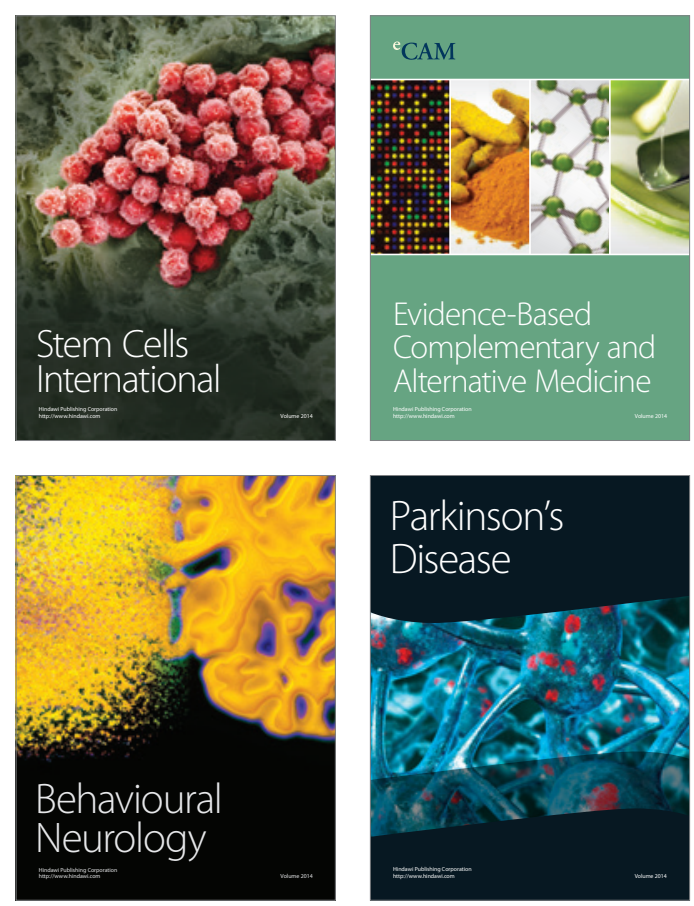

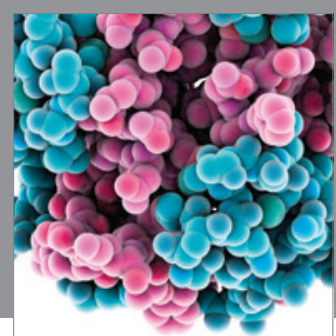

Journal of
Diabetes Research

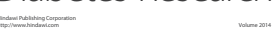

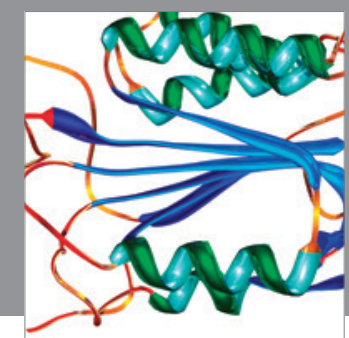

Disease Markers
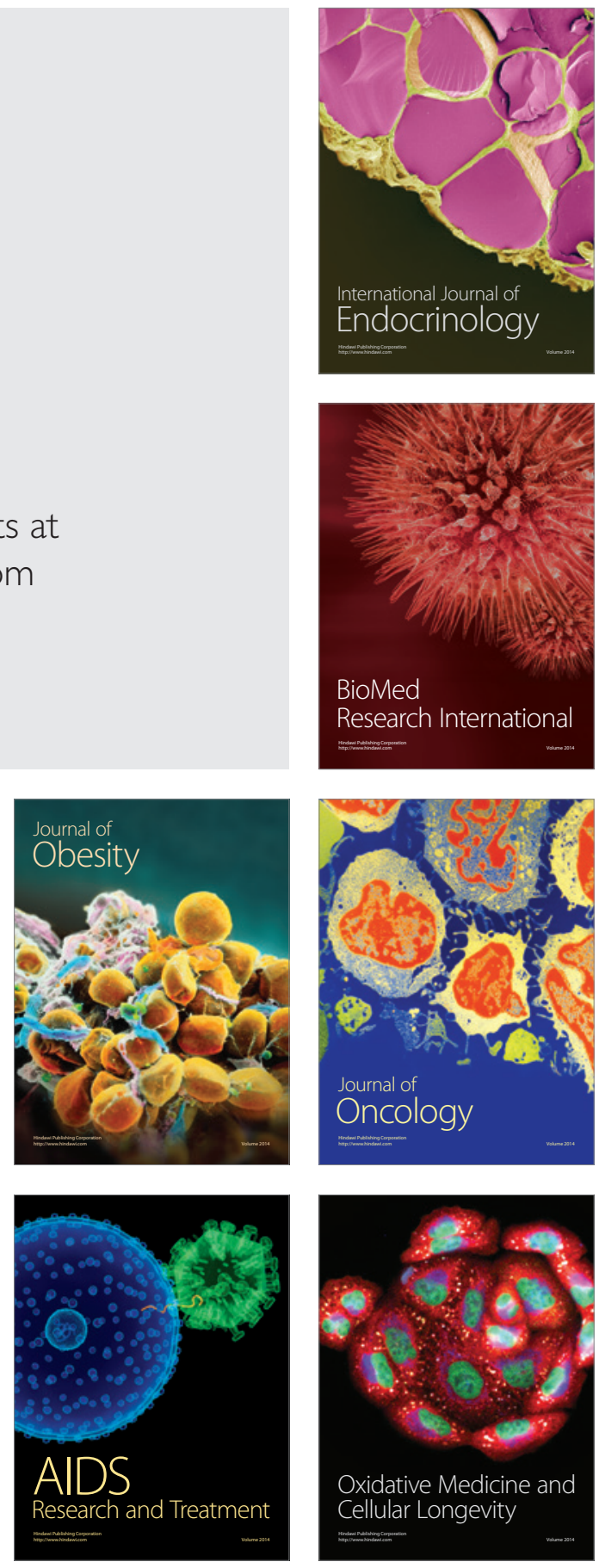